\title{
Chapter 13 \\ A Community-Based Risk Communication Approach on Low-Dose Radiation Effect
}

\author{
Naoki Yamano
}

\begin{abstract}
A community-based risk communication approach for risk and riskrelated factors regarding low-dose radiation has been started in 2013. In this approach, three groups that consist of local citizens, health nurse, midwife and nutritionist, and media reporters have been coordinated, and they discuss and examine a guidebook of health effects on low-dose radiation prepared by experts. Then they will revise the contents and expressions of the guidebook under expert's support by themselves. An improved guidebook implementing stakeholders' input will be expected through this process. In parallel to the community-based risk communication, an opinion survey has been conducted for the inhabitants of Tsuruga City in the Fukui prefecture to obtain people's cognition about ionizing radiation and risk on health effects. The inhabitants of about $43 \%$ show strong anxiety for radiation. They also show strong requirement for the risk criteria that should be specified by government/specialists. The current status and progress of the community-based risk communication approach are discussed, and a necessity of risk education regarding trans-science problem is presented.
\end{abstract}

Keywords Risk communication - Low-dose radiation - Fukushima nuclear accident $\bullet$ Public engagement

\subsection{Introduction}

Even now, after three and a half years or more from the Fukushima Daiichi nuclear accident, the health effects of low-dose ionizing radiation have become a national anxiety. Many activities of risk communication performed by the government are likely not successful though most inhabitants received estimated dose less than a few mSv.

Before the Fukushima accident, nuclear risk communication in Japan has been developed for public acceptance and improvement of nuclear power promotion understanding under the prerequisite that the safety is ensured. The risk

N. Yamano $(\bowtie)$

Research Institute of Nuclear Engineering, University of Fukui, 1-2-4 Kanawacho, Tsuruga,

Fukui 914-0055, Japan

e-mail: yamano_n@u-fukui.ac.jp

K. Kamae (ed.), Earthquakes, Tsunamis and Nuclear Risks,

DOI 10.1007/978-4-431-55822-4_13 
communication is expected as an effective method to regain public trust after the Fukushima accident. There is a good review for the historical background of risk communication in Japan [1]. However, risk communication for the health effects of low dose of ionizing radiation is not easy to perform because the low-dose effect has uncertainty including its sociological and psychological nature. Many specialists including radiation scientists, biologists, and health physicists have tried to explain the low-dose radiation effect to public, but it cannot be said that the public understanding through dialogue is effective because the specialists are not communication experts. The difficulties of risk communication for low-dose radiation have been reviewed by Kanda [2].

The author has started a new risk communication approach concerning health effects of low-dose radiation in 2013. This approach is intended to establish a community-based risk communication regarding low-dose radiation. In parallel to the community-based risk communication, an opinion survey has been done at September 2013 for inhabitants of Tsuruga City in the Fukui prefecture where 14 nuclear reactors are located in its vicinity. In Chap. 2, a summary of the opinion survey and the result is described. Chapter 3 describes the community-based risk communication approach and its progress. Chapter 4 will show insights and discussion through the community-based risk communication approach.

\subsection{Opinion Survey for Tsuruga Inhabitants}

The opinion survey was conducted at mid-September 2013 for 300 adult inhabitants of Tsuruga City in the Fukui prefecture. The aim of this survey is to obtain people's cognition about ionizing radiation and risk on health effects.

The survey method was a visit questionnaire placement method to obtain a sample number of 300 by assigning 15 people each in residential areas of 20 . Questions consisted of (1) the awareness about radiation and radioactivity, (2) the awareness about "risk" and food safety, and (3) the awareness about health effects of low-dose radiation exposure. In this study, the authors avoided direct questions concerning awareness of nuclear power. This indicates that it is not a survey questioning the pros and cons of nuclear power. Once people recognized it as a questionnaire relating to approval or disapproval of nuclear power, there is a possibility that influence of bias occurs in answer.

The detailed analysis of the opinion survey is described elsewhere [3].

In terms of risk cognition, Tsuruga inhabitants have the following thoughts about risk:

- There is a correlation between experience of risk education and knowing how to judge risk.

- There is a correlation between knowing the meaning of risk and judging own risk.

- There is a tendency that knowing how to judge risk leads a sense of security. 
- People do not recognize a risk cognition which has a trade-offs relation between hazard and benefit.

- There is no correlation between experience of risk education and judging own risk.

- The requirement of Tsuruga inhabitants for the risk acceptance criteria of low-dose radiation that should be specified by government/specialists does not depend on the degree of their knowledge about the risk information of radiation.

The results are obtained from the detailed analysis of the opinion survey, so we indicate the reference [3]. The tendency of these correlations about risk knowledge and recognition shows some people have experience of risk education in specific field, but the knowledge is not for radiation. It is clearly shown that inhabitants have no experience of risk education on low-dose radiation.

\subsection{Community-Based Risk Communication Approach}

In terms of low-dose radiation, most of the general public have few knowledge on what is a probabilistic (stochastic) effect on radiation exposure and the risk. The risk concept is not uniquely defined yet. Some scientists and engineers have recognized that risk is "probability $\times$ consequences." However, according to the ISO guidelines on risk management ISO31000:2009 [4], the definition is "effect of uncertainty on objectives." The International Risk Governance Council (IRGC) also defines risk as "an uncertain consequence of an event or an activity with respect to something that humans value" [5]. Such consequences can be positive or negative, depending on the values that people associate with them.

There is some confusion about risk concept among specialists in different area. Also, a consensus of the stochastic health effects of low-dose radiation has not been established among radiation scientists and biologists.

After the Fukushima Daiichi accident, many Japanese radiation scientists tried to explain the low-dose radiation effect to the general public. A lot of risk information on health effects of radiation has been explained to the general public using persuasive message based on epidemiologic study and the LNT (linear no-threshold) model. The general public has anxiety for radiation, so the question to scientists is simple such as "Am I safe? What is health impact to children and offspring?" However, the answer is not simple because of the uncertainty of scientific evidence from the epidemiologic study and the LNT hypothesis. It shows the health effects of low-dose radiation mean a trans-science problem.

General public is not familiar with the "probability." They also have heard different opinions for the "probability effect" of the low-dose radiation from some radiation scientists or commentators. So, the majority of the general public feels the scientists and commentators untrustworthy. In this situation, it means that the risk communication for low-dose radiation is not easy to perform because of lack of credibility of scientists. 


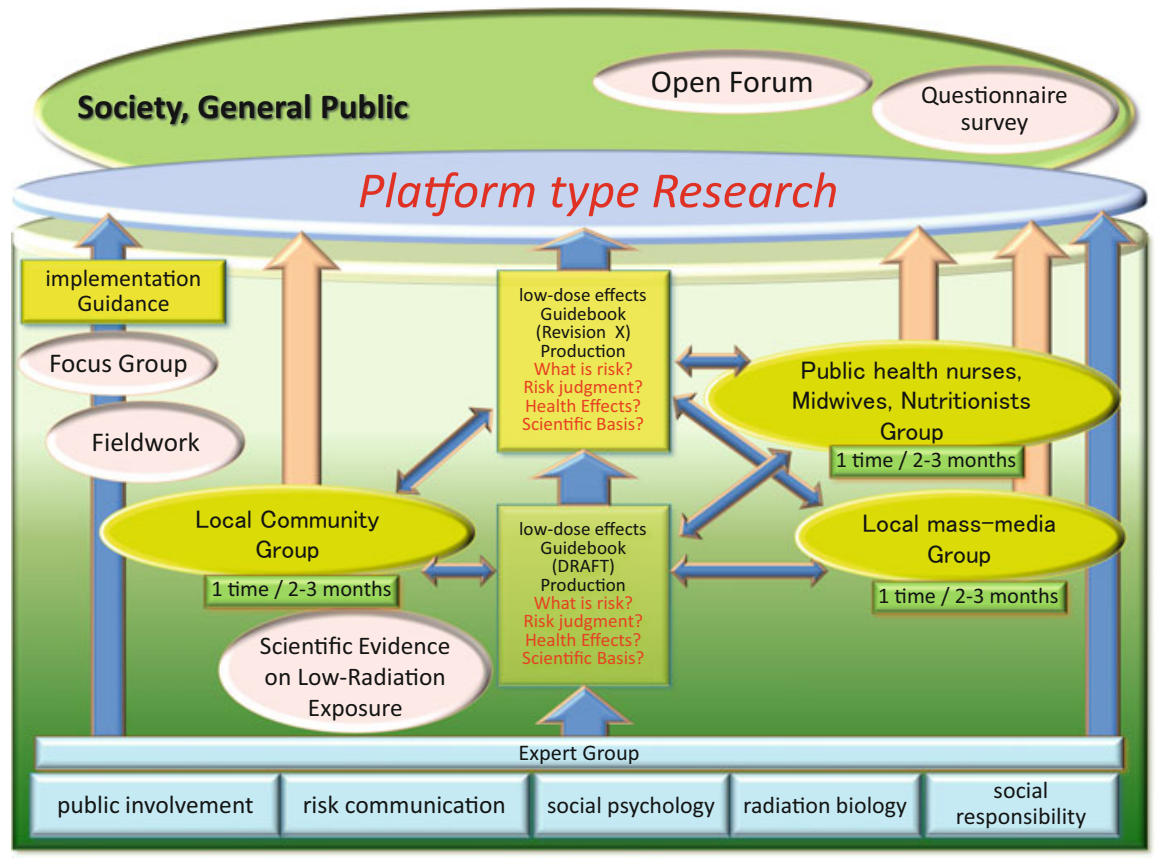

Fig. 13.1 A concept of the community-based risk communication approach

In general, a risk communication method which is designed for promoting stakeholders' willingness concerning "the right to know" and "the right to selfdetermination" is effective. There is diversity in the values of the general public. There are stakeholders who think that government/specialists should specify the risk criteria, but some stakeholders think strongly and want to judge it on their own based on the right to self-determination.

Based on this insight, a new risk communication approach concerning health effects of low-dose radiation has started in FY2013. A conceptual diagram of this approach is shown in Fig. 13.1. This approach is intended to establish public engagement of risk communication for risk and risk-related factors regarding low-dose radiation to promote the right to self-determination.

Firstly, a draft of guidebook of health effects on low-dose radiation was prepared in cooperation with experts of radiation biology, social psychology, risk communication, public involvement, and social responsibility. The draft consists of 46 pages including six chapters and references. The contents are Introduction (1), Concept of risk (2), Deterministic effects on radiation (3), What is the low dose? (4), Low-dose effects on radiation (5), and Conclusion (6).

Secondly, three groups have been coordinated in cooperation with Tsuruga inhabitants as follows:

- Local community inhabitants (12) who are members of female study group on nuclear power 
a

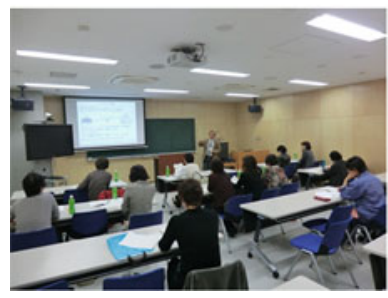

b

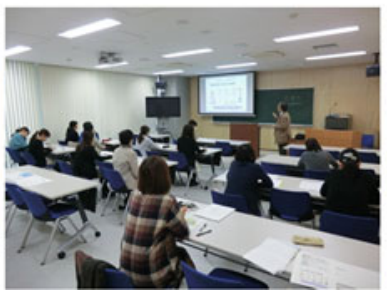

C

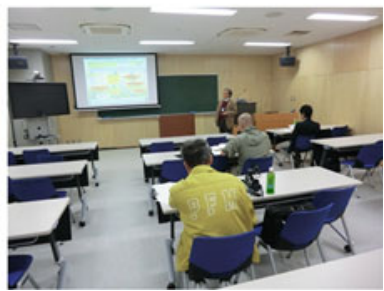

Fig. 13.2 Participants of workshop, (a) local community inhabitants; (b) health nurse, midwife, and nutritionist; (c) media reporters

- Health nurses, midwives, and nutritionists (12) who belong to the Tsuruga City health care center

- Media reporters (5) who belong to the Tsuruga press club

where the number in parenthesis indicates the number of participants in each group.

Participants of the three groups discuss and examine the draft of guidebook in workshop as shown in Fig. 13.2. Based on the dialogue and consultation, they will revise the contents and expressions of the guidebook under specialist's support by themselves. An improved guidebook implementing stakeholders' input will be expected through this process. The workshop has been held eight times in FY2013. In FY2014, creating the guidebook for beginner is in progress.

Besides that, we held public forums in the Tokyo metropolitan area and Tsuruga for the related researchers and general public. There were discussions on the opinion of the participants about the risk communication approach.

\subsection{Discussion}

Through the workshop using the draft of guidebook of health effects on low-dose radiation, the following opinions were obtained from participants:

- It is difficult to understand the risk concept, e.g., definition, probability, and uncertainty of risk.

- It is difficult to understand jargons such as DNA damage, repair mechanism, LNT model, EAR, ERR, Sv, Gy, or Bq.

- Epidemiologic study on health effects of low-dose radiation like CT scan is difficult to understand, but psychological impact after Chernobyl accident is well understood.

- There is too much information to understand. Avoid detailed contents, and fewer pages are favorable.

- It should change the order of the chapter because the risk concept is difficult.

- It is favorable to create a beginner's guidebook with fewer pages. 


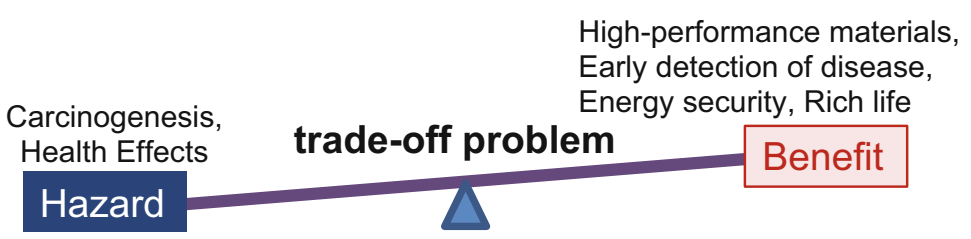

Fig. 13.3 Trade-offs relation between hazard and benefit on radiation risk

It seems that the general public does not easily understand the risk concept including definition and the risk cognition. Risk can be recognized as positive or negative depending on the values that people associate with them. If ionizing radiation is hazardous and has no benefit to people, they will not accept it whether it is low dose or not.

So, a relationship of trade-offs between hazard and benefit is important to understand radiation risk. Authors describe risk can be recognized as positive and negative, and the relationship between hazard and benefit as negative versus positive. We are trying to explain the risk trade-offs using a balance scale between (negative) hazard and (positive) benefit as shown in Fig.13.3.

Potential risk of low-dose ionizing radiation is "hazard" as cause of cancer, but it also has "benefit" like early detection of disease. The meaning of radiation risk and how to judge the risk on own is important to know. Most of the general public has no experience on learning about risk at education institutions. Some Tsuruga inhabitants have learned about risk at education institutions, but it seems that the risk education was for typical application, not for low-dose ionizing radiation. Even though they have knowledge on how to make typical risk judgment to other applications, they believe that the knowledge cannot be applicable to their health effects of ionizing radiation.

Through workshop, participants discussed with each other issues concerning the importance of understanding health effects of radiation and how to judge the radiation risk. They have understood that they should judge the risk of low-dose radiation on own whether the risk criteria specified by government/specialists are adequate or not. They also understood how to avoid or reduce the risk of artificial ionizing radiation.

\subsection{Conclusion}

A community-based risk communication approach concerning health effects of low-dose radiation has been described. The method is designed to promote stakeholders' willingness concerning not only "the right to know" but also "the right to self-determination."

The author conducted an opinion survey for Tsuruga inhabitants in order to obtain people's cognition about ionizing radiation and risk on health effects. The 
inhabitants of about $43 \%$ people show strong anxiety for radiation and have strong requirement for the risk criteria that should be specified by the government/specialists. It is believed that they are not satisfied with the current criteria of low-dose radiation. From the opinion survey, most of the public have no experience learning about the risk at education institution. However, it is found a tendency that knowing how to judge risk leads a sense of security. So, risk education for low-dose radiation seems to be able to reduce unnecessary anxiety.

The current status and progress of the community-based risk communication approach have been discussed. Through the community-based risk communication approach, participants have understood that they should judge the risk of low-dose radiation by their own, whether the risk criteria specified by government/specialists are adequate or not. Participants also understood how to avoid or reduce risk from artificial radiation. An improved guidebook implementing stakeholders' input will be expected through this process. The present method will be effective to public understanding about risk of low-dose radiation.

Acknowledgments This work was supported by JSPS KAKENHI Grant Number 25420902.

Open Access This chapter is distributed under the terms of the Creative Commons Attribution Noncommercial License, which permits any noncommercial use, distribution, and reproduction in any medium, provided the original author(s) and source are credited.

\section{References}

1. Kinoshita T (2014) Short history of risk communication in Japan. J Dis Res 9:592-597

2. Kanda R (2014) Risk communication in the field of radiation. J Dis Res 9:608-618

3. Shinoda Y, Yamano N (2015) Survey of Tsuruga inhabitants concerning radiation and its risks. Trans Atomic Energy Soc Jpn 14(2):95-112, doi:10.3327/taesj.J14.018. [in Japanese]

4. ISO 31000:2009, Risk management - principles and guidelines. 2009. International Organization for Standardization

5. Renn O (2005) White paper on risk governance, towards an integrative approach. International Risk Governance Council. Available at http://www.irgc.org/ 